\title{
A BROWNIAN POPULATION MODEL
}

\author{
B.S. LAKSHMI
}

\begin{abstract}
We consider a population model in which there is no information about the births and deaths and so can be modelled by a random process. We come to the interesting conclusion that even though births and deaths have equal probability,the population itself steadily increases towards the maximum sustainable level.
\end{abstract}

\section{INTRODUCTION}

In its simplest idealized form, the Random-Walk Problem can be formulated as follows [4.A person starts walking on a street.Each step he takes is of length $l$.The person walks to the left or to the right and the direction of each step is completely independent of the preceding step. Thus the probability of its being to the right is $p$ while the probability of its being to the left is $q=1-p$. Choosing the $\mathrm{X}$ axis to lie along the street so that $x=0$ is the position of the point of the origin,the location of the man along the $\mathrm{X}$-axis must be of the form $x=m l$,where $\mathrm{m}$ is an integer .The question of interest is :after the man has taken $\mathrm{N}$ steps, what is the probability of his position being located at the position $x=m l$ ?

Instead of considering a man taking steps we use the language of physics and think of a particle performing successive steps or displacements in one dimension.After a total of $\mathrm{N}$ steps each of length $l$, the particle is located at $x=m l$ where $m$ is an integer lying between $-N \leq m \leq N$. If the particle moves $n_{1}$ steps to the right and $n_{2}$ steps to the left,so that $N=n_{1}+n_{2}$, the probability $P_{N}(m)$ of finding the particle at the position $x=m l$ after $\mathrm{N}$ such steps is given by the binomial distribution

$$
P_{N}(m)=\frac{N !}{n_{1} ! n_{2} !} p^{n_{1}} q^{n_{2}}
$$

Date: Received: 18 May 2010

(C) 2010 N.A.G.

Key words and phrases. population models. 
Clearly the above equation is also the probability of the particle taking $n_{1}$ steps to the right. The mean of taking $n_{1}$ steps to the right is given by

$$
\overline{n_{1}}=N p
$$

and the mean of taking $n_{2}$ steps to the left is given by

$$
\overline{n_{2}}=N q
$$

The dispersion $\overline{\left(\Delta n_{1}\right)^{2}}$ : can be calculated to be

$$
\overline{\left(\Delta n_{1}\right)^{2}}=N p q
$$

The quantity $\overline{\left(\Delta n_{1}\right)^{2}}$ is quadratic in the displacement.Its square root, the rootmean-square deviation

$$
\Delta^{*} n_{1} \equiv\left[\overline{\left(\Delta n_{1}\right)^{2}}\right]^{1 / 2}
$$

is a linear measure of the width of the range over which $n_{1}$ is distributed.A good measure of the relative width of the distribution is ,in particular if $p=q=1 / 2$,

$$
\frac{\Delta^{*} n_{1}}{\overline{n_{1}}}=\frac{1}{\sqrt{N}}
$$

Since $\mathrm{m}$ is the net displacement $m=n_{1}-n_{2}=2 n_{1}-N$,we obtain by taking averages,

$$
\overline{(\Delta m)^{2}}=4 N p q
$$

In particular if $p=q=1 / 2$

$$
\overline{(\Delta m)^{2}}=N
$$

Our main concern in this paper is not that of a man walking in a random manner,nor that of a moving particle but the random process of birth and death,with birth and death having equal probability.

\section{A Brownian Population Model}

We consider a population $(\mathrm{P}(\mathrm{t}))$ whose growth rate would be,following (1.2), proportional to $\sqrt{P(t)}$

$$
\frac{d P(t)}{d t}=K \sqrt{P(t)}
$$

where $\mathrm{K}$ is some proportionality constant.If we integrate (2.1), we get

$$
P(t) \propto K^{2} t^{2}
$$

This shows that the Population grows indefinitely with time.As such it is not a realistic model.So we modify (2.1), taking the cue from the logistic equation [5]. If $\mathrm{M}$ is the maximum sustainable population, $\mathrm{K}$ cannot be independent of the population, as in the Logistic Model. Thus

$$
\frac{d P}{d t}=r(M-P) \sqrt{P} \quad r>0
$$

Using standard techniques we solve $(\overline{2.2})$, to obtain

$$
\left.\frac{\sqrt{M}+\sqrt{P}}{\sqrt{M}-\sqrt{P}}=\mathbf{C} e^{\sqrt{M} r t} \quad \text { (C is a constant }\right)
$$


(2.3) gives an interesting and realistic solution.It shows that as time $t \rightarrow \infty$, the population, $P(t)$ smoothly approaches the maximum sustainable population M.Then even though births and deaths take place at random,with equal probability,the population increases.

\section{A Predator-Prey Example with Brownian Effects}

This model finds a parallel in nature as was found in the experiments of mathematical biologist G.F.Gause.In these experiments, the population was composed of two species of Protozoa, one of which,Didinium nasatum feeds on the other Paramecium caudatum.In all of Gause's experiments, the Didinium quickly destroyed the Paramecium and then died of starvation.This situation cannot be modelled by the usual predator-prey system of equations,

$$
\begin{aligned}
& \frac{d x}{d t}=a x-b x y-e x^{2} \\
& \frac{d y}{d t}=-c y+d x y-f y^{2}
\end{aligned}
$$

$(\mathrm{x}(\mathrm{t})$ representing the population of the prey and $\mathrm{y}(\mathrm{t})$ representing the population of the predator, a,b,c,d,e,f being constants) since no solution of (3.1) with $x(0) y(0) \neq 0$ can reach $\mathrm{x}=0$ or $\mathrm{y}=0$ in finite time.

The reason for this is that the Didinium are a special and atypical type of predator.On the one hand they are ferocious attackers and require a tremendous amount of food; a Didinium demands a fresh Paramecium every three hours. On the other hand the Didinium do not perish from an insufficient supply of Paramecium..They continue to multiply, but give birth to smaller offspring. Thus the system of equations (3.1)does not accurately model the interaction of the Didinium and Paramecium. A better model in this case is the system of differential equations

$$
\frac{d x}{d t}=a x-b \sqrt{x} y, \quad \frac{d y}{d t}= \begin{cases}d \sqrt{x} y, & x \neq 0, \\ -c y, & x=0\end{cases}
$$

(3.2) can be solved and it can be shown that every solution $x(t), y(t)$ with $x(0)$ and $y(0)$ positive reaches $\mathrm{x}=0$ in finite time[1]. From (3.2) we can see that there is a parallel with the non-predator model $(2.2)$,in the term $\sqrt{x}$ which gives the random element.

\section{Discussion}

There are situations in nature where it is impossible to make any definitive statements about the population and its environment,for example the available food ,or a hostile climate,or a sudden unpredictable natural calamity.In other words the process is now random rather than deterministic. The model proposed could be useful for a probabilistic population growth,taking into consideration randomness of events.Finally we observe that the solution (2.3) does not easily lend itself to the case where the maximum sustainable population $M$ can be periodic. This latter case has been shown to lead to a whole new theory [2, 3]. This would be a topic for future work. 


\section{REFERENCES}

[1] M. Braun, Differential equations and their applications, Springer-Verlag,4th ed., 1993.

[2] B.S. Lakshmi, Oscillating Population Models, Chaos, Solitons and Fractals, 16 (2003) 183-186.

[3] E.C. Pielou, Mathematical Ecology, John Wiley New York. 1976.

[4] F. Reif, Fundamentals of statistical and thermal physics, McGraw Hill, Singapore, 1988.

[5] S. Werner, S. Weigelhofer and L. Kenneth, Ordinary Differential Equations and Applications, Albion Publishing Chichester, 1998.

Jawaharlal Nehru Technological University, College of Engineering, KukatPALLY,HYDERABAD ,INDIA

E-mail address: bslakshmi2000@yahoo.com. 